\title{
Reduced Waiting Times Using a Fast Switching Dual- Polarization DDQPSK Receiver in a Packet Switched Network
}

\author{
Anthony J. Walsh ${ }^{(1,2)}$, James Mountjoy ${ }^{(3)}$, Anthony Fagan ${ }^{(3)}$, Colm Browning ${ }^{(1)}$, Andrew D. Ellis ${ }^{(1,4)}$, \\ Liam P. Barry ${ }^{(1)}$ \\ (1) Rince Institute, Dublin City University, Glasnevin, Dublin 9, Ireland, anthony.walsh66@mail.dcu.ie \\ (2) Tyndall National Institute, University College Cork, Lee Maltings, Cork, Ireland. \\ (3) School of EECE, University College Dublin, Dublin 4, Ireland. \\ (4) Aston Institute of Photonic Technologies, School of Engineering and Applied Science, Aston \\ University, Birmingham B4 7ET, England.
}

Abstract In this paper, we demonstrate a fast switching dual polarization DDQPSK packet switched
receiver with very short waiting times. The system employs $\mathrm{m}^{\text {th }}$ power DDQPSK decoding for high
frequency offset tolerance, and Stokes parameter estimation for robust polarization demultiplexing.

\section{Introduction}

There is an ever-present demand for higher data rates through the optical internet ${ }^{1}$. At the same time, the non-linear Shannon capacity of singlemode fibre is being reached ${ }^{2}$. One way to improve data rates further in the network is to improve temporal utilization of network resources by performing optical packet switching (OPS) and spectral utilisation through coherent detection of polarisation multiplexed modulation formats.

OPS networks typically use fast switching tunable lasers in order to change the transmitter's wavelength to a particular receiver's wavelength to achieve routing. After a switching event, when a tunable laser changes wavelength, there is typically a large frequency transient, as seen in $^{3}$, which will give a large frequency offset transient between the transmitting laser and receiver local oscillator (LO). This can result in errors for coherent transmission systems if the frequency offset compensation algorithm has a limited range, since the frequency offset transient may drift outside the algorithm's range. This issue can be resolved using $\mathrm{m}^{\text {th }}$ power doubly differential quadrature phase shift keying (DDQPSK) which has a very large frequency offset tolerance ${ }^{4}$, with a similar algorithm discussed in ${ }^{5}$.

If dual-polarization is to be implemented as well in an OPS system, e.g. dual-polarization quadrature phase shift keying (DP-QPSK), there will be an issue with the convergence times of the widely used constant modulus algorithm $(\mathrm{CMA})^{3}$, as well as issues with reliability due to its singularities, and efforts to address this have been undertaken ${ }^{3}$. In this paper, the CMA algorithm will be replaced by a different polarization demultiplexing algorithm which estimates the Stokes parameters of the received constellation using a least means squares (LMS) method $^{6}$ which offers shorter convergence times. We experimentally demonstrate a novel dual-polarization DDQPSK packet receiver with far shorter waiting times than previously reported, successfully receiving data only $\sim 30$ ns after a wavelength switch.

\section{Robust DP-DDQPSK Packet Receiver}

The LMS Stokes parameter estimation scheme described $i^{6}$ works on the basis that the $s_{1}, s_{2}$ and $s_{3}$ parameters for DP-mPSK form a flat surface/lens in the 3D space of $\left(s_{1}, s_{2}, s_{3}\right)$. By calculating the slope of this plane, the polarization rotation angles of the Jones matrix can be determined, allowing for a polarization demultiplexing Jones matrix to be calculated. The outputs from the polarization diverse coherent receiver at specific time samples, $I_{x}$, $Q_{x}, I_{y}$ and $Q_{y}$ can be used to calculate complex field variables $E_{x}$ and $E_{y}$, and to calculate $s_{1}, s_{2}, s_{3}$ via the equations ${ }^{6}$ :

$$
\begin{gathered}
E_{x}=(1 / a) \cdot\left(I_{x}+j Q_{x}\right) \\
E_{y}=(1 / b) \cdot\left(I_{y}+j Q_{y}\right) \\
s_{1}=\frac{1}{2} \operatorname{Re}\left(E_{x}\left(E_{x}\right)^{*}-E_{y}\left(E_{y}\right)^{*}\right) \\
s_{2}=\frac{1}{2} \operatorname{Re}\left(\left(E_{x}\right)^{*} E_{y}+E_{x}\left(E_{y}\right)^{*}\right) \\
s_{3}=\frac{1}{2} \operatorname{Re}\left(-j\left(E_{x}\right)^{*} E_{y}+j E_{x}\left(E_{y}\right)^{*}\right)
\end{gathered}
$$

where $a$ and $b$ are conversion efficiencies. Here we assume $a=1$ and $b=1$ without loss of generality. The normal vector to the plane of $\left(s_{1}\right.$, $\left.s_{2}, s_{3}\right)$, is a $1 \times 3$ matrix with elements $\left(a_{1}, a_{2},-1\right)$, where, in this paper, $a_{1}$ and $a_{2}$ are estimated using an LMS equation which averages $(2 n+1)$ samples bracketing the sample of interest:

$$
\left(\begin{array}{l}
a_{1} \\
a_{2}
\end{array}\right)=\left(\begin{array}{ll}
\sum_{-n}^{n} s_{1}^{2} & \sum_{-n}^{n} s_{1} s_{2} \\
\sum_{-n}^{n} s_{1} s_{2} & \sum_{-n}^{n} s_{2}^{2}
\end{array}\right)^{-1}\left(\begin{array}{l}
\sum_{-n}^{n} s_{1} s_{3} \\
\sum_{-n}^{n} s_{2} s_{3}
\end{array}\right)
$$


The inverse Jones matrix is calculated using:

$$
\begin{gathered}
\Delta \phi=\tan ^{-1}\left(a_{2},-1\right) \\
\theta=\frac{1}{2} \tan ^{-1}\left(a_{1}, \sqrt{1+a_{2}^{2}}\right) \\
M_{\text {inverse }}=\left(\begin{array}{cc}
\cos (\theta) \cdot e^{j \Delta \phi / 2} & \sin (\theta) \cdot e^{-j \Delta \phi / 2} \\
-\sin (\theta) \cdot e^{j \Delta \phi / 2} & \cos (\theta) \cdot e^{-j \Delta \phi / 2}
\end{array}\right)
\end{gathered}
$$

where $\tan ^{-1}(p, q)$ is the two argument arctan with $p$ as the cos input and $q$ as the sin input. $M_{\text {inverse }}$ is the compensating matrix. Eq. (7) to Eq. (9) are as given in ${ }^{6}$, whilst Eq. (6) was derived by reducing the conventional $3 \times 3$ matrix to a $2 \times 2$ matrix, utilizing the fact that the $\left(s_{1}, s_{2}, s_{3}\right)$ plane passes through the origin. The algorithm is applied three times in parallel with the parameters $\left(s_{1}, s_{2}, s_{3}\right)$ cyclically interchanged in Eq. (6), to avoid ambiguities in case that the DPmPSK 2D surface is parallel to one of the $\left(s_{1}, s_{2}\right)$, $\left(s_{2}, s_{3}\right)$, or $\left(s_{3}, s_{1}\right)$ planes. The rotated $\left(s_{1}, s_{2}, s_{3}\right)$ axis system which gives the largest determinant of the $2 \times 2$ matrix before inversion in Eq. (6) is the axis system which is selected.

It is clear that since there is no initialization of the LMS algorithm, and since it is feed-forward, the convergence time will only be limited by the number of summation terms used. This results in consistent convergence times unlike the CMA method, which gives variable convergence times depending on the input state of polarisation ${ }^{3}$.

Furthermore, since the LMS algorithm's compensation matrix is never singular $^{6}$, two independent outputs are always produced.

In addition to using this more robust polarization demultiplexing algorithm, the $\mathrm{m}^{\text {th }}$ power DDQPSK algorithm used in $^{4}$ was used here to provide very large frequency offset tolerance (the frequency offset is only limited by the bandwidth of the receiver electronics). This algorithm has been previously demonstrated for wavelength switched single polarisation signals, resulting in reduced switching times due it having a larger frequency offset range than the typical $\mathrm{m}^{\text {th }}$ power single differentially encoded QPSK (SDQPSK) scheme ${ }^{4}$. This was achieved with less than $1 \mathrm{~dB}$ penalty compared with $\mathrm{m}^{\text {th }}$ power SDQPSK, and doubly differential decoding also protects against cycle slip error propagation. In previous DP-QPSK schemes (some of which employ differential encoding) maximum waiting times to correctly receive data are approximately $288 \mathrm{~ns}$ with the aid of training symbols $^{7}$, and $410 \mathrm{~ns}$ using 25 initial test CMA steps to initialize the system ${ }^{3}$. However, here we show that waiting times can be improved by an order of magnitude ( 30ns) by using both the LMS algorithm and doubly differential decoding.

\section{Experimental Setup}

The experimental setup is shown in Fig. 1 below. The transmitter was a Sampled-Grating Distributed Bragg Reflector (SG-DBR) laser switching between two wavelengths $(1541.2 \mathrm{~nm}$ and $1547.4 \mathrm{~nm}$ ). The SG-DBR laser was on each wavelength for $\sim 500 \mathrm{~ns}$ giving it a switching frequency of $1 \mathrm{MHz}$. The transmitter was connected to a $90 / 10$ coupler where the $10 \%$ output was used to measure frequency offset transients and the $90 \%$ output was connected, via a polarization controller $(P C)$ and a polarization beam splitter (PBS), to a pol-mux-IQ modulator. The PBS slow axis output went into the modulator. The $10 \mathrm{Gbit} / \mathrm{s}$ modulating signals $I_{x}, Q_{x}, I_{y}$ and $Q_{y}$ came from the data and data (bar) outputs of a pattern generator, with delays introduced to decorrelate the data signals. The delays introduced were 10 bits between $I_{x}$ and $Q_{x}, 25$ bits between $I_{y}$ and $Q_{y}$, and 31 bits between $Q_{x}$ and inverted $I_{y}$. The delays were set so that it resulted in two different $E_{x}$ and $E_{y}$ data patterns, allowing for polarization rotations to be identified. $10 \%$ of the modulator output was amplified, filtered and sent to an oscilloscope for eye-diagram monitoring, whilst the remaining $90 \%$ was input to a noise loading stage where attenuated amplified spontaneous emission noise was added to the modulated signal through a 50/50 coupler, with one output from the coupler connected to an optical spectrum analyzer (OSA) for optical signal to noise ratio (OSNR) measurements, performed in a similar way to 4 , and the other output connected to an erbium doped fibre amplifier (EDFA), $2 \mathrm{~nm}$ filter and $P C$ before reaching the signal port of a polarization diverse coherent receiver. A tunable laser source (TLS) at $1541.2 \mathrm{~nm}$ went through a $P C$ and then a polarizer before reaching the LO port of the coherent receiver. The receiver outputs were recorded by a 50Gsample/s real- time oscilloscope.

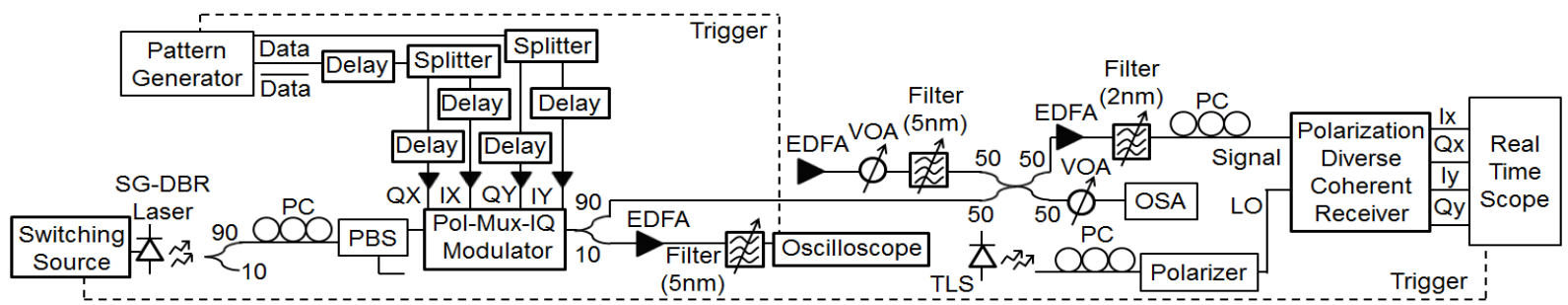

Fig. 1: Experimental setup of BER versus OSNR measurement system for packet switched DP-DDQPSK. 
Digital signal processing on the received $X$ and $Y$ fields was performed offline. Firstly, the fields had offsets removed, amplitudes normalized, and timing imbalances equalized. The optimum sampling point was found by choosing the sample with the largest average amplitude of $E_{x}$ and the received fields were then downsampled. Then, polarization demultiplexing was performed using either the LMS method described above or the CMA method. After this, the modified $\mathrm{m}^{\text {th }}$ power frequency offset method described $i^{4}$ was used, followed by the $\mathrm{m}^{\text {th }}$ power phase estimation stage. A hard decision was then made on the constellation, after which doubly differential decoding was performed on the hard decision symbols. These symbols were converted to bits using Gray coding and BER values were calculated by direct error counting. The lowest BER polarisation was arbitrarily designated as the $\mathrm{X}$-polarisation. The average BER over both polarizations was calculated for each OSNR value using approximately $7.2 \times 10^{5}$ bits.

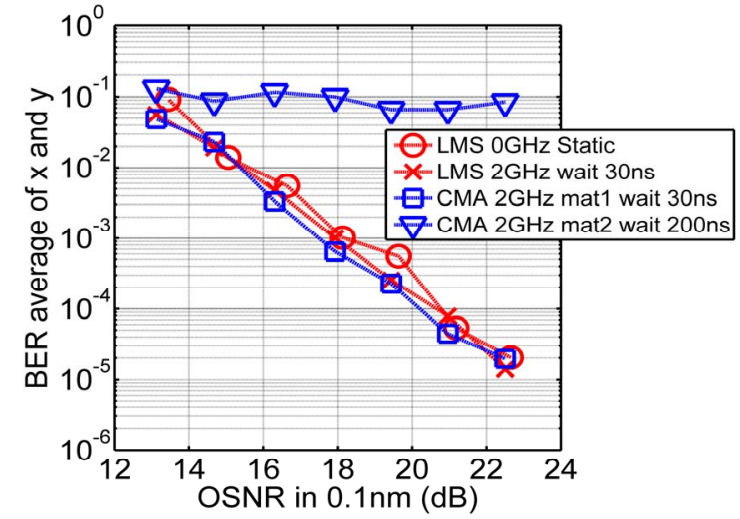

Fig. 2: BER versus OSNR for pol-mux-DDQPSK using LMS or CMA with a static and switching laser for different waiting times and for different steady-state frequency offsets.

\section{Results}

Fig. 2 shows the BER versus OSNR for polmux-DDQPSK using $\mathrm{m}^{\text {th }}$ power with either the LMS or CMA methods, in static and switching scenarios. Different waiting times after the tunable laser switches wavelength were used, and different steady-state frequency offsets were used. The CMA had an initial demultiplexing matrix of either mat1 or mat2 (both unitary matrices). As seen in Fig. 2, the CMA converged reasonably well with mat 1 but is unable to decode data correctly with mat 2 , even with a large waiting time of 200ns due to the presence of singularities. The LMS, by contrast, can work in a fully blind manner with respect to the polarization state of the input signal, which is important in an OPS scenario where packets from different network nodes will have different states of polarization. All curves, except the high BER one, can achieve BER values of $10^{-3}$ at an OSNR of approximately $18.2 \mathrm{~dB}$. The required waiting time of $30 \mathrm{~ns}$ is mainly due to the 101 averaging terms used in the frequency offset estimation (averaging reduces the impact of noise on the estimate) and this results in averaging of frequency transients at the start of the packet. In Fig. 2, the static and switching LMS curves are overlapping, demonstrating similar performance. In addition, Fig. 2 shows that pol-mux-DDQPSK can tolerate frequency offsets of $2 \mathrm{GHz}$ in the switching cases.

\section{Conclusions}

It has been shown for the first time that by using $\mathrm{m}^{\text {th }}$ power DDQPSK and LMS polarization demultiplexing, a DP-DDQPSK packet-switched system can be implemented with very short waiting times required after a wavelength switch in order to correctly receive data. The short waiting time presented, of around $30 \mathrm{~ns}$, is an order of magnitude better than previous work, with improved robustness to both input state of polarization and large frequency offsets.

\section{Acknowledgements}

We would like to acknowledge funding from Science Foundation Ireland under grant the numbers 09/IN.1//2653 and 12/RC/2276.

\section{References}

[1] D. J. Bishop et al., "Energy Efficient Networking: Avoiding a Future Energy Crunch," Proc. MILCOM, p. 2047, San Jose (2010).

[2] A. D. Ellis et al., "Approaching the Non-Linear Shannon Limit", J. Lightwave Technol., Vol. 28, no. 4, p. 423 (2010).

[3] R. Maher et al., "Widely Tunable Burst Mode Digital Coherent Receiver with Fast Reconfiguration Time for 112 Gb/s DP-QPSK WDM Networks", J. Lightwave Technol., Vol. 30, no. 24, p. 3924 (2012).

[4] A. J. Walsh et al., "Reduced OSNR Penalty for Frequency Drift Tolerant Coherent Packet Switched Systems Using Doubly Differential Decoding", Proc. OFC, Th4D.8, San Francisco (2014).

[5] H. Ishikawa et al., "Frequency-Offset Compensation for MPSK Differential Detection by Using Double Differential Encoding", Electronics and Communications in Japan, Part 1, Vol. 78, no. 1, p.62 (1995).

[6] B. Szafraniec et al., "Polarization demultiplexing in Stokes space," Optics Express, Vol. 18, no. 17, p. 17928 (2010).

[7] J. Gripp et al., "Wavelength-Tunable Burst-Mode Receiver with Correlation-Based Polarization Separation", Proc. ECOC, Th.2.A.3, London (2013). 